\title{
Electrical Percolation during Codeposition of Fe and Si Clusters by a Dual Source PGCCD System
}

\author{
Nobuyuki Shinohara*, Ryoji Katoh and Kenji Sumiyama \\ Department of Materials Science and Enginnering, Nagoya Institute of Technology, Nagoya 466-8555, Japan
}

Random assemblies of Fe and Si clusters have been prepared using a dual source plasma gas condensation cluster deposition system and their conductivities were measured for various compositional ratios. The size distributions of Fe and Si clusters are obtained by transmission electron microscopy (TEM) observations and the volume fraction of Fe clusters, $f_{\mathrm{Fe}}$, is estimated from the chemical analysis and packing fraction of cluster assemblies. Electrical conductivity, $\sigma$, of Fe and Si cluster assemblies obeys a power law of $f_{\mathrm{Fe}}$. The dramatic change at around $f_{\mathrm{Fe}}=f_{\mathrm{c}}$, indicates that the percolation of Fe clusters takes place at the threshold value, $f_{\mathrm{c}}=0.16$. It is consistent with the Links-Nodes-Blobs model calculated for a three dimensional percolation network. [doi:10.2320/matertrans.MBW200708]

(Received October 17, 2007; Accepted January 23, 2008; Published March 12, 2008)

Keywords: cluster assembly, iron cluster, silicon cluster, transmission electron microscopy, conductivity, nano-composite material, percolation

\section{Introduction}

Anomalous and functional properties of nanostructurecontrolled materials are interesting topics in materials science and technology. They have been usually made from supersaturated solution via low temperature heat treatment. In such precipitation processes, the particle size and interparticle distance cannot be adjusted independently owing to the materials balance. ${ }^{1)}$ Assembling of clusters is an alternative method for fabricating nanostructured materials because nanometer sizes of clusters are the same as elemental units of materials' structures and functionalities. The size uniformity, stability against oxidation and suppression of inter-cluster aggregation are requisite to utilize the sizedependent characteristics of clusters.

Using a plasma-gas-condensation cluster deposition (PGCCD) system, we have prepared mono-disperse size transition metal clusters whose mean diameters, $d$, range between 5 and $15 \mathrm{~nm}$ with the standard deviation less than $10 \%$ of $d .^{2)}$ When such clusters are continuously deposited on a substrate in the PGCCD system, their numbers are increased while their sizes are maintained. With further increasing the deposition time they agglomerate to form geometrical and electrical networks. These features can be well understood as percolation phenomena.

In the next step, preparation and deposition of different kinds of clusters are interesting challenges. They are analogous to alloying of two elements in metallurgy, which has given a lot of high functional alloys and displayed the prosperity of materials. For this purpose we developed a PGCCD system which had two glow discharge sources: one glow discharge was a dc mode and the other an rf or dc discharge mode: two sputtering chambers were divided by a simple plate which could separate two glow discharges and two kinds of vapor atoms sputtered out of the targets. When the separation plate was inserted between two glow-discharge chambers, a mixture of $\mathrm{Fe}$ and $\mathrm{Si}$ clusters was obtained on a substrate: small Fe clusters were distributed at

*Graduate Student, Nagoya Institute of Technology, Corresponding author, E-mail: shinohara@ss.emat.nitech.ac.jp random, while the Si clusters were aggregated to form large secondary particles. Without inserting the separation plate, on the other hand, core-shell clusters were obtained: Fe core were surrounded by small Si crystallites. ${ }^{3,4)}$ These results indicate that $\mathrm{Fe}$ and $\mathrm{Si}$ clusters do not merge to form alloyand compound-clusters though the chemical affinity of $\mathrm{Fe}$ and $\mathrm{Si}$ is so high as to form very stable intermetallic compounds in the equilibrium phase diagram. ${ }^{5,6)}$

In order to perform better control of cluster-preparation and -deposition processes we have recently developed a dual source PGCCD system. In this study, we codeposited Fe and Si clusters using this new PGCCD system. We carried out transmission electron microscope (TEM) observations of Fe and Si clusters codeposited on TEM microgrids. We also did in-situ observations of electrical conductivity during codeposition processes of $\mathrm{Fe}$ and $\mathrm{Si}$ clusters on glass substrates to discuss their percolation behaviors.

\section{Experimental}

Figure 1 shows a newly developed dual source PGCCD system, which consists of two DC glow discharge sources installed in the completely separated sputtering chambers,

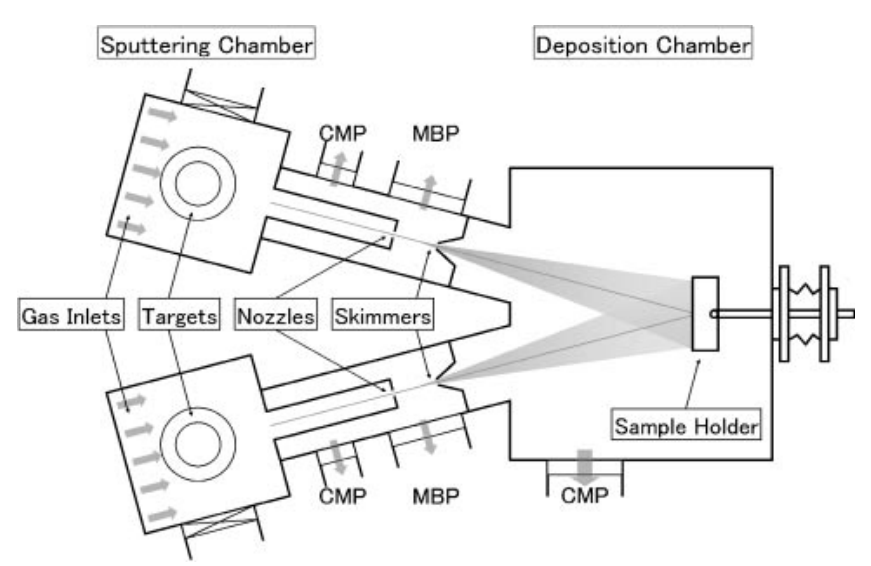

Fig. 1 A schema of the newly developed plasma gas condensation cluster deposition apparatus. 
two cluster growth rooms, intermediate evacuation chambers and a deposition room. All of them were first evacuated down to $10^{-5} \mathrm{~Pa}$ by turbo molecular and composite molecular pumps, TMP and CMP. In the PGCCD system, which was a hollow cathode type, two $\mathrm{Fe}$ and two Si plates of $80 \mathrm{~mm}$ in diameter and $5 \mathrm{~mm}$ in thickness were set on two target holders, where $\mathrm{Fe}$ was $99.9 \%$ pure and $\mathrm{Si}$ was n-type doped of $10 \mathrm{ppm} \mathrm{B} \mathrm{for} \mathrm{DC} \mathrm{sputtering.} \mathrm{Since} \mathrm{magnetic} \mathrm{field} \mathrm{was}$ applied perpendicular to these targets and its intensity at the perimeter was higher than the one at the center, the metal plates were rather uniformly and efficiently sputtered out in contrast to non-uniform target erosion of conventional magnetron sputtering.

For glow discharge sputtering TMPs were shut down and a large amount of Ar gas (99.9999 vol.\%) was independently introduced through gas-inlets with adjusting mass flow meters, while growth rooms were evacuated by a mechanical booster pump $\left(800 \mathrm{~m}^{3} / \mathrm{h}\right)$ to eject the Ar gas, sputtered atoms and formed clusters through the small nozzle of intermediate evacuation chambers. Here, the Ar gas flow rates were $6.7 \times 10^{-6} \mathrm{~m}^{3} / \mathrm{s}$ : the corresponding Ar gas pressure of the sputtering chambers 1 and 2 was $270 \mathrm{~Pa}$, while that of the deposition chamber was $0.3 \mathrm{~Pa}$ by the TMP evacuation. The input electric powers for the glow discharge were as follows: the electric current was 1.00 A, and the voltage between 300$350 \mathrm{~V}$ for the Fe targets and 400-450 V for the Si targets. The thickness of the deposited cluster assembly, $t$, was measured with a stylus intrument (SURFCM1400D). In this study we first prepared and deposited only $\mathrm{Fe}$ and $\mathrm{Si}$ clusters on substrates by operating one of the dual glow discharge sources, and then $\mathrm{Fe}$ and $\mathrm{Si}$ clusters simultaneously on a substrate by operating the dual glow discharge sources.

The morphology, size and structure of $\mathrm{Fe}, \mathrm{Si}$ and $\mathrm{Fe} / \mathrm{Si}$ clusters were observed using a transmission electron microscope (TEM; Hitachi Co., HF-2000) operating at $200 \mathrm{kV}$. TEM images were observed for specimens on TEM grids at the initial deposition stage and selected area electron diffraction patterns for those at the late deposition stage. The cluster size distributions were determined from digitized images recorded by a slow-scan charge-coupled device camera for the area of $350 \times 350 \mathrm{~nm}^{2}$ using the imageanalysis software (Image-Pro PLUS: Media Cybernetics).

During the deposition of clusters an electrical current was measured between two stripe electrodes on a glass substrate (the widths of $2 \mathrm{~mm}$ and the separation distance of $0.5 \mathrm{~mm}$ ) with application of an electric voltage of $0.1 \mathrm{~V}$ using a digital multimeter (Keithley2400,236).

Compositional ratios, $\mathrm{Fe} / \mathrm{Si}$ were determined by energy dispersive X-ray (EDX) analyzers installed both in TEM and in a scanning electron microscope (SEM) (Hitachi S-4700) operating at $5 \mathrm{kV}$, where the $\mathrm{O}$ content was neglected owing to the quantitative resolution limit.

\section{Result}

Figure 2(a) shows a bright-field TEM image of Fe clusters and (c) the size distribution histogram estimated from Fig. 2(a), indicating that the cluster sizes are distributed between 5 and $15 \mathrm{~nm}$ : the average Fe cluster size is about $9.3 \mathrm{~nm}$ and the standard deviation is about $27 \%$. As shown in (a)

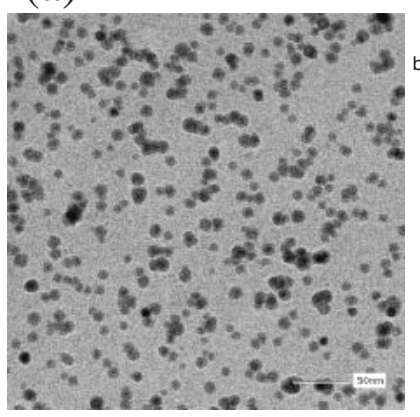

(c) (b)
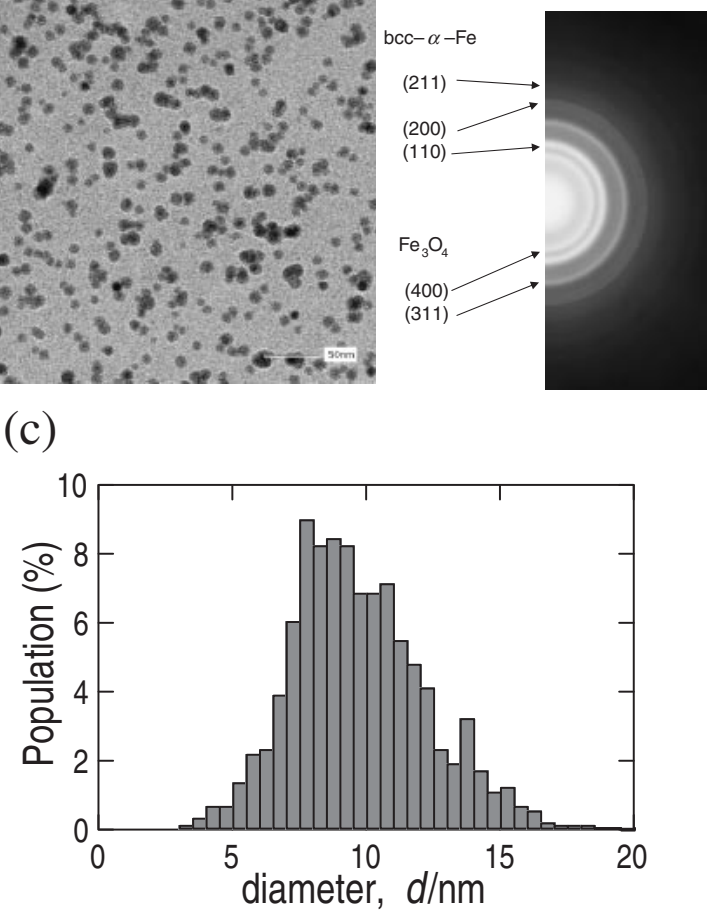

Fig. 2 (a) Bright-field transmission electron microscope (TEM) image and (b) electron diffraction (ED) pattern and (c) size distribution of Fe clusters deposited on TEM grids.

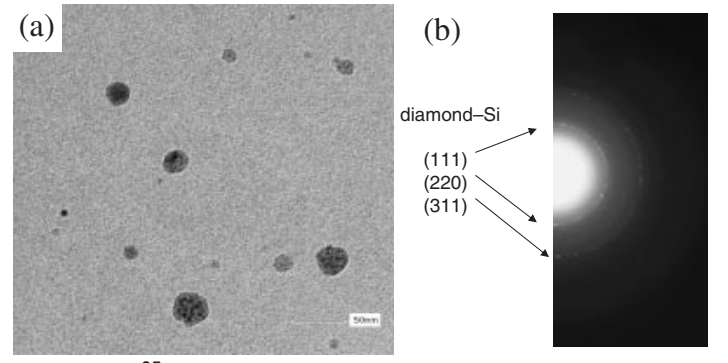

(c)
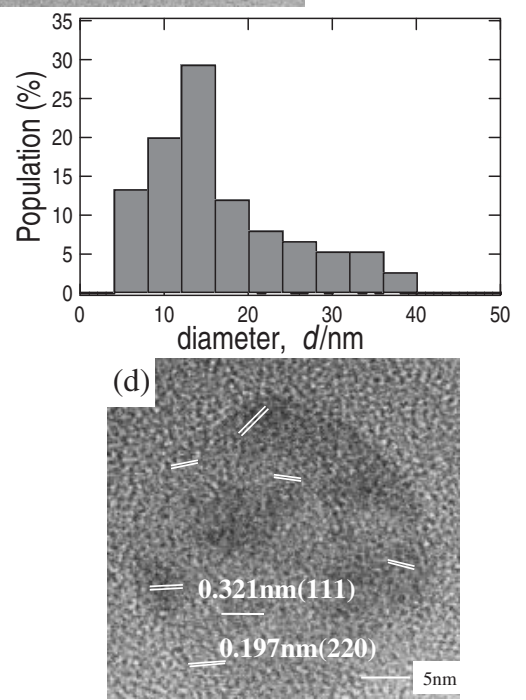

Fig. 3 (a) Bight-field transmission electron microscope (TEM) image, (b) electron diffraction (ED) pattern, (c) size distribution and (d) highresolution TEM image of Si clusters deposited on TEM grids. 
(a)

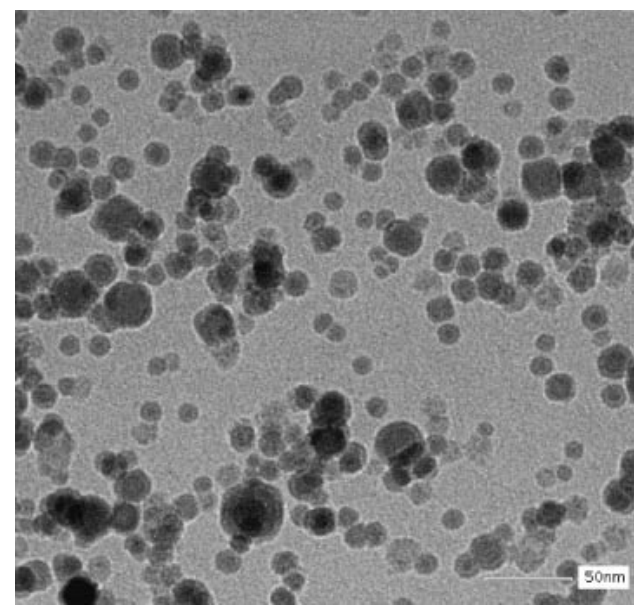

(b)

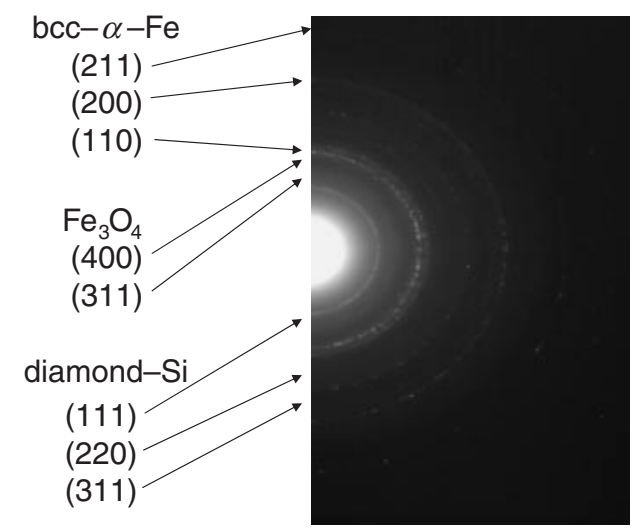

Fig. 4 (a) Bight-field TEM image and (b) electron diffraction (ED) pattern of Fe and Si cluster codeposited on a TEM grids.

Fig. 2(b) (the selected area diffraction patterns of the $\mathrm{Fe}$ clusters), bcc $(\alpha-\mathrm{Fe})$ and $\mathrm{Fe}_{3} \mathrm{O}_{4}$ type diffraction rings are detected, indicating the partial oxidation of Fe clusters.

Figure 3(a) shows a bright-field TEM image of Si clusters and Fig. 3(c) the size distribution histogram estimated from Fig. 3(a), indicating that the cluster sizes are widely distributed between 5 and $40 \mathrm{~nm}$ : the average $\mathrm{Si}$ cluster size is about $16.5 \mathrm{~nm}$ and the standard deviation is about $51 \%$. Figure 3(a) also shows that the large $\mathrm{Si}$ particle consists of small crystalline particles. As shown in Fig. 3(b) (the selected area diffraction patterns of the Si clusters), diamond type diffraction rings are detected whereas their widths are very broad. The high-resolution TEM image of $\mathrm{Si}$ clusters shown in Fig. 3(d) demonstrates that a $\mathrm{Si}$ cluster is a secondary aggregate and contains much smaller crystallites.

Figures 4(a) and (b) show a bright-field TEM image and the selected area diffraction patterns of $\mathrm{Fe}$ and $\mathrm{Si}$ clusters codeposited under the same conditions as those for Figs. 2 and 3. In Fig. 4(a) the size distribution of these clusters are remarkable, whereas $\mathrm{Fe}$ and $\mathrm{Si}$ clusters are not markedly coalesced each other. In Fig. 4(b), the electron diffraction rings indicated by arrows can be indexed as bcc- $\alpha$-Fe, $\mathrm{Fe}_{3} \mathrm{O}_{4}$ and diamond-type $\mathrm{Si}$ phases being similar to those of $\mathrm{Fe}$ and $\mathrm{Si}$ clusters in Figs. 2(b) and 3(b). However, no clear diffraction ring corresponding to intermetallic compounds, $\mathrm{Fe}_{3} \mathrm{Si}, \mathrm{FeSi}_{2}$, etc. cannot be detected.

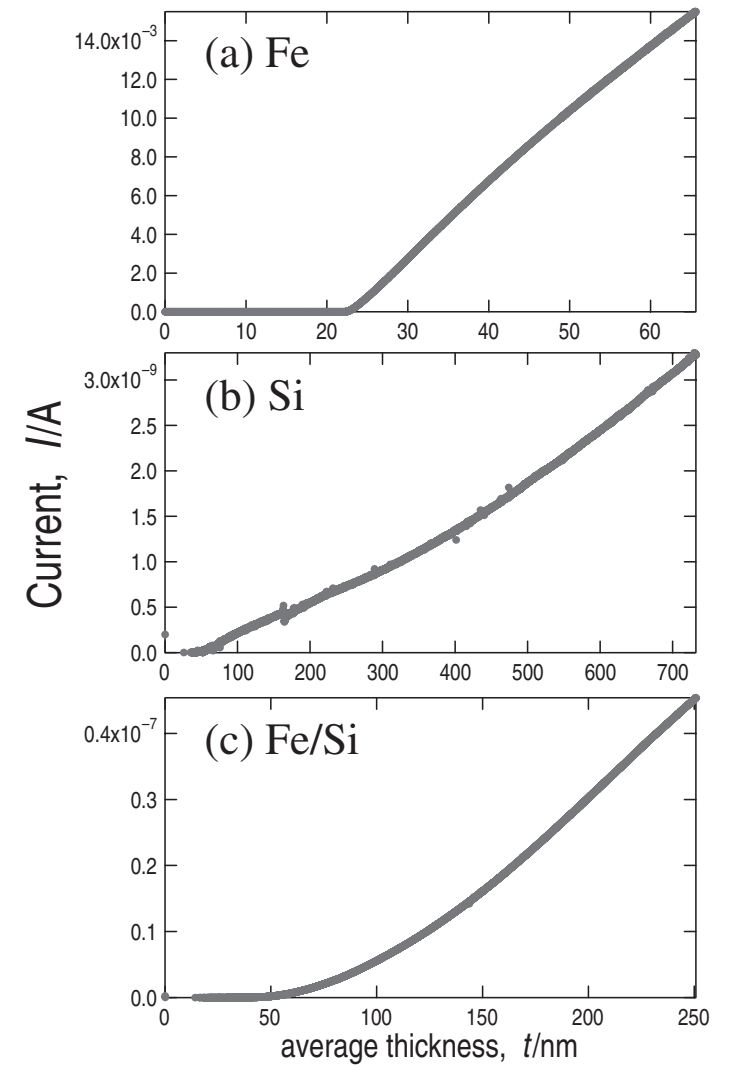

Fig. 5 Electric current (I) changes against the average thickness in (a) Fe, (b) $\mathrm{Si}$ and (c) $\mathrm{Fe}$ and $\mathrm{Si}$ cluster assemblies, where the average $\mathrm{Fe}$ composition in (c) is about 60 at\% $\mathrm{Fe}$.

Figures 5(a), (b) and (c) show changes of electrical current $(I)$ as a funcition of the average deposition thickness of $\mathrm{Fe}, \mathrm{Si}$ and $\mathrm{Fe}$ and $\mathrm{Si}$ clusters, $t$, which were observed between two electrodes on substrates during deposition of $\mathrm{Fe}$ and $\mathrm{Si}$ clusrters, and codeposition of Fe and Si clusters. For deposition of Fe clusters (Fig. 5(a)), $I$ is negligibly small for $t<23 \mathrm{~nm}$. It rather suddenly starts to increase at $t=24 \mathrm{~nm}$ and nonlinearly increases for $t>24 \mathrm{~nm}$, suggesting a two dimensional electrical percolation. With further increasing $t, I$ linearly increases with increasing $t$ for $t>$ $35 \mathrm{~nm}$. Similarly for deposition of Si clusters in Fig. 5(b), $I=0$ A for $t<51 \mathrm{~nm}$ and it starts to increase at $t=52 \mathrm{~nm}$, while the non-linear change is observed for $t=52 \sim 400 \mathrm{~nm}$ and the linear change for $t>400 \mathrm{~nm}$. For codeposition of $\mathrm{Fe}$ and $\mathrm{Si}$ clusters whose compositional ratio, $\mathrm{Fe} / \mathrm{Si}=$ $60 / 40$ in Fig. 5(c), $I=0 \mathrm{~A}$ for $t<32 \mathrm{~nm}$ and it start to increase at $t=33 \mathrm{~nm}$. Two step changes are detected: a non-linear one for $t=33 \sim 150 \mathrm{~nm}$ and a linear one for $t>150 \mathrm{~nm}$.

Taking account of the thickness and area of cluster assemblies on a substrate, we estimated the electrical conductivity, $\sigma$. Table 1 shows $\sigma$ values of $\mathrm{Fe}$ and $\mathrm{Si}$ cluster assemblies in comparison with those of bulk specimens. $\sigma$ of the Fe cluster assembly is 50 times smaller than that of bulk specimens, probably due to their porous stacking, while $\sigma$ of $\mathrm{Si}$ cluster is 1200 times larger than that of bulk specimens due to the heavy doping of Si targets. Here, it is worth to emphasize that $\sigma$ of the Fe cluster assembly is a six order larger than $\sigma$ of the Si cluster assembly. 
Table 1 Electrical conductivity, $\sigma(1 / \Omega \mathrm{m})$, of Fe cluster- and Si clusterassemblies together with their bulk specimens.

\begin{tabular}{ccc}
\hline & cluster assembly & bulk \\
\hline $\mathrm{Fe}$ & $1.92 \times 10^{5}$ & $9.93 \times 10^{6}$ \\
\hline $\mathrm{Si}$ & $3.04 \times 10^{-1}$ & $2.52 \times 10^{-4}$ \\
\hline
\end{tabular}

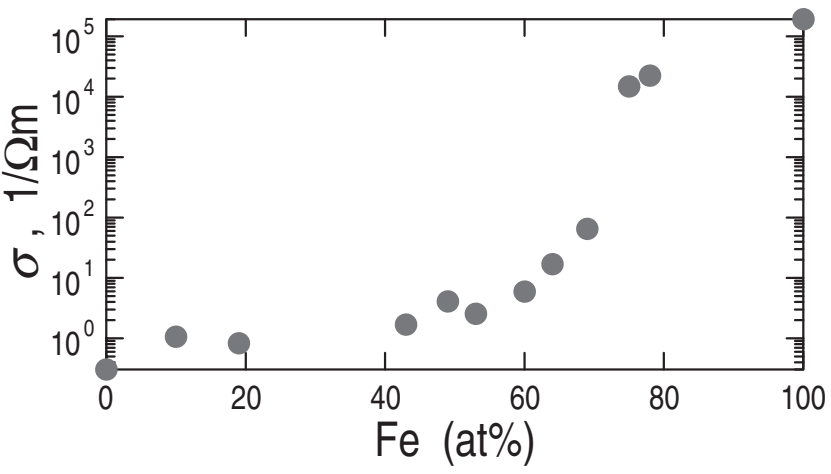

Fig. 6 Electrical conductivity, $\sigma$, of $\mathrm{Fe}$ and $\mathrm{Si}$ cluster assemblies as a function the average Fe composition.

Figure 6 shows $\sigma$ versus the $\mathrm{Fe}$ composition (at $\%$ ) in codeposited assemblies of $\mathrm{Fe}$ and $\mathrm{Si}$ clusters, whose average thicknesses were more than $200 \mathrm{~nm}$. Here, the Fe compositions were estimated by the EDX analyzer in SEM. Since the central positions of Fe and Si cluster beams were not so well coincided, the dispersion in $\mathrm{Fe}$ compositions among a specimen was almost 5 at $\%$. $\sigma$ is very low, being less than $0.5 \Omega^{-1} \cdot \mathrm{m}^{-1}$ for $\mathrm{Fe} / \mathrm{Si}<60 / 40$ while it rapidly increases for $\mathrm{Fe} / \mathrm{Si}>60 / 40$.

\section{Discussion}

Figures 2(c) and 3(c) reveals that the size distribution of $\mathrm{Si}$ clusters is wider than that of Fe clusters and Figs. 2(a) and 3(d) reveals that the primary crystalline size of Si clusters are much smaller than that of $\mathrm{Fe}$ clusters. These results are consistent with the Gibbs and Thomson criterion for nucleation of a small substance: ${ }^{7)}$ Si clusters are more stable than Fe clusters in their small size ranges because the surface energy of $\mathrm{Si}$ is about a half of that of $\mathrm{Fe}$. In the growth rooms, such small primary Si crystallites collide with each other and agglomerate to form secondary particles. ${ }^{8)}$ Since they are oxidized after exposing to ambient atmosphere, the very broad diffraction rings of Si clusters can be ascribed both to the small crystalline sizes and to formation of deficient silicon mono-oxides that have amorphous-like structures.

Fe and Si clusters formed in high Ar gas atmosphere of the present dual source PGCCD system are well thermalized via many time collisions with $\mathrm{Ar}$ atoms in the growth rooms. After passing through the nozzles and skimmers, these clusters do not collide with each other so often and impinge on the substrate because the vacuum condition becomes much better. This speculation is supported by the low deposition rate of clusters (the maximum rate is $1.1 \mathrm{~nm} / \mathrm{sec}$.). The velocities of $10 \mathrm{~nm}$ size Fe and Si clusters are the same order of magnitude as that of Ar gas flow velocity, which is at most a sound velocity when it pass through the nozzle

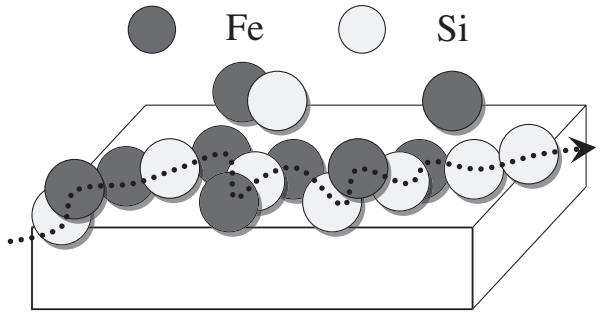

(a) two dimensional connection

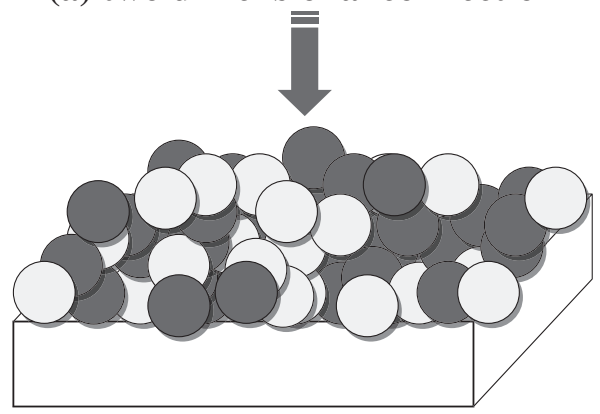

(b) three dimensional connection

Fig. 7 Schematic images of (a) a two dimensional electrical percolation threshold and (b) a three dimensional connection of clusters assemblies.

between the growth room (its $\mathrm{Ar}$ gas pressure is about $200 \mathrm{~Pa}$.) and the intermediate evacuation room (its $\mathrm{Ar}$ pressure is about $2 \mathrm{~Pa}$.) Since the kinetic energy of impinging clusters onto the substrate surface is about a few hundred $\mathrm{eV}$, the clusters can migrate on the substrate. ${ }^{1,9)}$ However, the clusters of about $10 \mathrm{~nm}$ in diameter contain several ten thousands of atoms and the kinetic energy per atom is an order of $10^{-3}-10^{-2} \mathrm{eV}$, being much smaller than the cohesive energy of $\mathrm{Fe}, \mathrm{Si}$ and $\mathrm{Fe}-\mathrm{Si}$ compounds. Even if such clusters collide with each other and impinge on the substrate, their fragmentation and merging cannot be expected at $\mathrm{Fe}$ and $\mathrm{Si}$ cluster interfaces, and $\mathrm{Fe}$ and $\mathrm{Si}$ clusters randomly coexist on the substrate, as shown in Fig. 4(a).

As shown in Figs. 5(a), (b) and (c), the $I$ versus $t$ curves reveal the two step changes. At the initial stage $I$ is negligibly small, indicating that the two electrodes are not electrically connected by a network of clusters. The sudden increase in $I$ at a certain value of $t$, suggests a threshold of two dimensional electrical percolation, whose schematic image is shown in Fig. 7(a). The data of non-linear $t$ dependence of $I$ have been fitted to the following scaling law,

$$
I \propto\left(t-t_{c}\right)^{\mu},
$$

where $t_{c}$ is the critical thickness and $\mu$ the critical exponent, being the fitting parameters. ${ }^{10)}$ In the previous studies on the deposition of uniform size Co clusters, we discussed the size dependence of the percolation phenomena and interpreted by the soft percolation model. ${ }^{11)}$ In the present study, however, we do not study a detailed size-dependence in the two dimensional percolation because the size uniformities of $\mathrm{Fe}$ and $\mathrm{Si}$ clusters are not so good as those of Co clusters prepared by the single source PGCCD system.

Then, we pay attention to the $t$ linear dependence of $I$ for heavily stacked specimens in Fig. 5 because it clearly indicates a three dimensional connection of these clusters, 


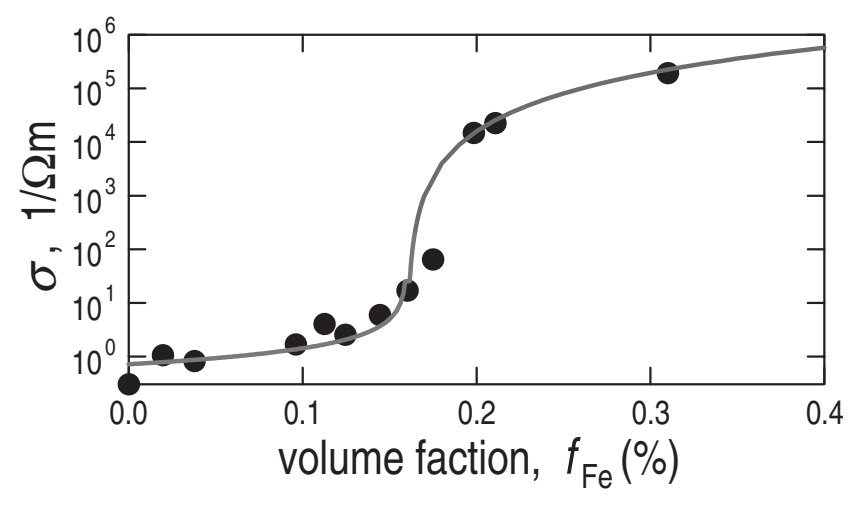

Fig. 8 Electrical conductivity, $\sigma$, versus the volume fraction of Fe clusters $\left(f_{\mathrm{Fe}}\right)$ in $\mathrm{Fe}$ and $\mathrm{Si}$ cluster assemblies. The closed symbols are the experimental results and the curve is the calculated one by eq. (2) and (3).

whose schematic image is shown in Fig. 7(b). Since Fe is a metal and $\mathrm{Si}$ is a semiconductor, the electrical conductivity of $\mathrm{Fe}$ and Si cluster assemblies are so different as mentioned in Table 1. Figure 6 also demonstrates that $\sigma$ changes dramatically at around the deposition ratio of $\mathrm{Fe} / \mathrm{Si}=60 / 40$ : a $\mathrm{Si}$ cluster network predominates for $\mathrm{Fe} / \mathrm{Si}<60 / 40$, while a $\mathrm{Fe}$ cluster network for $\mathrm{Fe} / \mathrm{Si}>60 / 40$.

In order to understand the three dimensional percolation in the present codeposited assemblies of Fe and Si clusters, we introduce the following power law proposed for the mixed assembly of metal and insulator particles: ${ }^{12-14)}$

$$
\begin{array}{ll}
\sigma \propto\left(f-f_{c}\right)^{\alpha} & \left(f>f_{c}\right) \\
\sigma \propto\left(f_{c}-f\right)^{\beta} & \left(f<f_{c}\right),
\end{array}
$$

where $f$ is the volume fraction of metal particles, $f_{\mathrm{c}}$ the critical value of $f$, and $\alpha(>0)$ and $\beta(<0)$ the critical exponents for discussing the conductivity of these mixed assemblies. Taking into account of the random distribution in the electrical current channel for $f>f_{\mathrm{c}}$, eq. (2) is rewritten by the following Links-Nodes-Blobs formula:

$$
\sigma \propto\left(f-f_{c}\right)^{(d-2) v+1}
$$

where $d$ is a assembly dimension of two different particles ( $d=3$ for the present case) and $v$ the critical exponent. Since the packing density 30-35\% for the assemblies of $\mathrm{Cr}$, Co, and Fe clusters prepared by previous PGCCD systems, ${ }^{15,16)}$ we assume $p=0.3$ for estimating the volume fraction of $\mathrm{Fe}$ clusters, $f_{\mathrm{Fe}}$. Figure 8 shows the $\sigma$ values as a function of $f_{\mathrm{Fe}}$, derived from Fig. 6. As shown in this figure, the model curves, choosing $f_{\mathrm{c}} \approx 0.15, \beta \approx-0.7$ for $f<f_{\mathrm{c}}$ and $v \approx 0.9$ for $f>f_{\mathrm{c}}$ are well fitted to the estimated results. The value of $f_{\mathrm{c}}$ is close to the theoretically calculated one, $f_{\mathrm{c}}=0.15$, for a three-dimensional random lattice, while it is much different from $f_{\mathrm{c}}=0.44$ for two dimensional random lattice. ${ }^{17)}$ Such a drastic change in $\sigma$ is a typical percolation phenomenon, demonstrating that metallic Fe clusters and semiconducting Si clusters are loosely stacked and no marked alloying behavior is induced in these $\mathrm{Fe}$ and Si cluster assemblies.

\section{Summary}

Using a newly developed dual source PGCCD system, $\mathrm{Fe}$ and $\mathrm{Si}$ clusters have been independently or simultaneously deposited on substrates. In the TEM images of codeposited assemblies of $\mathrm{Fe}$ and $\mathrm{Si}$ clusters, bcc and diamond-type phases coexist, where neither alloying nor merging of these two kinds of clusters is observed. In-situ observations of electrical conduction during deposition of these clusters demonstrate two steps changes from two dimensional to three dimensional conduction channel (networks) formations, i.e., the characteristic features of electric percolation phenomena. The conductivity versus volume fraction of $\mathrm{Fe}$ clusters plot also reveals the percolation phenomenon for assemblies of metal $\mathrm{Fe}$ and semiconductor $\mathrm{Si}$ clusters, being well interpreted by the Links-Nodes-Blobs model.

\section{Acknowledgments}

This work has been supported by Intellectual Cluster Project supported by the Ministry of Education, Science, Culture and Sports, Japan, Aichi Prefecture, Nagoya City and Aichi Science and Technology Foundation.

\section{REFERENCES}

1) K. Sumiyama, T. Hihara, D. L. Peng and S. Yamamuro: Encyclopedia of Nanoscience and Nanotechnology, Vol. 10 Transition Metal Nanocluster Assemblies, ed. H. S. Nalwa, (American Scienctific Publishers, 2004) p. 471-507.

2) S. Yamamuro, K. Sumiyama and K. Suzuki: J. Appl. Phys. 85 (1999) 483-489.

3) R. Katoh, T. Hihara, D. L. Peng and K. Sumiyama: Appl. Phys. Lett. 87 (2005) 252501-252503.

4) R. Katoh, T. Hihara, D. L. Pemg and K. Sumiyama: Appl. Phys. Lett. 82 (2003) 2688-2690.

5) F. R. de Boer, R. Boom, W. C. Mattens and A. R. Miedema: Cohesion in Metals-Transition Metal Alloys (North-Holland, Amsterdam, 1989) p. 662 .

6) T. B. Massalski, J. L. Murray, L. H. Bennett, H. Baker and L. Kacprzak: Binary Alloy Phase Diagrams 2nd ED. (American Society of Metals, Metals Park, Ohio) p. 1711.

7) For example C. Kittel and H. Kroemer: Thermal Physics 2nd ED (W. H. Freeman and Company, New York, 1980) p. 295.

8) R. Katoh, T. Hihara, D. L. Peng and K. Sumiyama: J. Appl. Phys. 100 (2006) 034308-1-034308-7.

9) P. Jensen: Rev. Mod. Phys. 71 (1999) 1695-1735.

10) D. Stauffer: Introduction to Percolation Theory, (Taylor \& Francis, 1985).

11) S. Yamamuro, K. Sumiyama, T. Hihara and K. Suzuki: J. Phys.: Condens. Matter 11 (1999) 3247-3257.

12) A. S. Skal and B. I. Shklovskii: Sov. Phys. Semicond. 8 (1975) 10291032.

13) P. G. de Gennes: J. Physique Lett. 37 (1976) L1-L2.

14) H. E. Stanley and A. Coniglio: Percolation, Structure and Process, ed. by G. Deutscher, R. Zallen and J. Adler, (Hilger, London, 1983) p. 101.

15) S. Yamamuro, K. Sumiyama, T. Kamiyama and K. Suzuki: J. Appl. Phys. 86 (1999) 5726-5732.

16) D. L. Peng, T. Hihara, K. Sumiyama and H. Morikawa: J. Appl. Phys. 92 (2002) 3075-3083.

17) H. Scher and R. Zallen: J. Chem. Phys. 53 (1970) 3759-3761. 